\section{ON A QUASI-ISOCHRONOUS B FACTORY IN THE PEP TUNNEL*}

\author{
S. HEIFETS \\ Stanford Linear Accelerator Center \\ Stanford Universitv, Stanford, CA 9.4309
}

\section{INTRODUCTION}

Isochronous, or quasi-isochronous 'torage rings with very small momentum ompaction factor $\alpha$ for various applications have been discussed recently [1] and studied in more details by C. Pellegrini and D. Robin [2] (this paper contains other references). This approach looks very promising for the B-Factory design. A small momentum compaction factor reduces the bunch length at the given $R F$ voltage. A small bunch length allows, in principle, reduction of the beta function at the interaction point (IP) with a corresponding increase of the luminosity. However, achieving this is not easy due to difficulties with local chromatic effects at the IP. more severe background problems in the detector, and enchanced adverse effects of the beam-beam interaction. Although these problems can be addressed, they require substantial changes in the present design. It is worth noting that shorter bunches would also have larger energy losses and larger energy spread. due to wake field excitation and the smaller disruption parameter reducing the enhancement factor. But energy losses scale with the bunch length $\sigma_{z}$ as $\sigma_{z}^{-1 / 2}$, remaining small compared with the losses due to synchrotron radiation. The disruption parameter is small in the present design anyway; Hence, these effects do not cause additional problems.

It was suggested that an isochronous ring may allow some reduction of the $\mathrm{RF}$ voltage. Reduction of the $\mathrm{RF}$ voltage is limited by the synchrotron losses and the beam loading. The amplitude of the field in a cavity $V_{c}$ driven at the $\mathrm{RF}$ frequency $\omega$ is $[3]$

$$
V_{c} e^{i(\phi-\xi)}=\left[V_{g}-V_{b} e^{i \xi}\right] \cos (\psi),
$$

where $V_{g}$ and $V_{b}$ are the voltages driven by a generator (klystron) and the beam with de component $I_{0}$ correspondingly,

$$
V_{g}=\frac{2}{1+\beta} \sqrt{P_{i} R_{a} \beta}, \quad V_{b}=\frac{R_{a} I_{0}}{2(1+\beta)},
$$

$\psi$ is detuning angle $\tan \psi=-(\delta \omega / \omega) Q_{L}, \phi$ is the phase of the voltage $V_{c}$ in a cavity and $\xi$ is the phase of the current in respect to the phase of the generator,
OCT

$R_{a}$ is the accelerator shunt impedance, and $\beta$ is the coupHng c18gqient. The accelerating voltage $V_{a}=V_{c} \cos (\psi-\xi)$ depends on the energy losses and defines the power $P_{i}$ of the generator. Consider detuning of the order of the revolution frequency spread in a bunch. Then $\psi$ is of the order of $\xi$ for a normal momentum compaction factor $\alpha$ and $\psi \ll \xi$ if $\alpha$ is reduced in a quasi-isochronous ring. In the latter case $\psi$ may be omitted, and the stability to the beam loading depends (also very weakly) only on $\xi$, related to a bunch length. Hence, the voltage $V_{c}$ and power $P_{i}$ are independent of $\alpha$. The present CDR design [4] uses parameters which already correspond to the threshold of the beam loafing stability and the optimum coupling coefficient $\beta$.

Dependence of the momentum compaction factor $\alpha$ on the relative energy $\delta=\Delta E / E$

$$
\alpha=\alpha_{1}+\alpha_{2} \delta
$$

adds a new nonlinear term to the equation of the synchrotron oscillations. The ratio of the new nonlinear term to the usual nonlinear term due to the nonlinearity of the potential well

$$
\frac{4 \alpha_{2} \cot \left(\phi_{s}\right)}{q \alpha_{1}^{2} \nu_{s}}
$$

increases for small $\alpha_{1}$ as $\alpha_{2} \alpha_{1}^{-3 / 2}$. The ratio is large for the $\alpha_{1}$ 's we are interested in.

A small compaction momentum factor results in low synchrotron tune $\nu_{s}$. This affects the structure of the synchro-betatron resonances driven by the chromaticity, dispersion in the RF cavities, nonlinearities of the lattice, and beambeam interaction. The width of the resonances decreases as some fractional power of $\nu_{s}$, while the distance between the resonances decreases as $\nu_{s}$. This means that for small $\nu_{s}$ there is a tendency for the resonances to overlap, giving stochastic motion. However, the resonances are very weak and can be wiped out by the synchrotron damping or, for a very small momentum compaction factor, they can merge without damaging the beam stability. The problem may be worth studying more carefully in future. The energy spread of the synchrotron frequencies may also have a positive effect on the beam stability.

We also considered fluctuations of the length of the orbit due to the fluctuations of the locations of the points where photons are radiated. This (usually neglected) noise gives a very small effect unless the reduction of $\alpha_{1}$ compared to a normal value is large compared to $\nu_{s} / N_{c}$, where $N_{c}$ is the number of cells per turn (periodicity of the dispersion function).

\section{ISOCHRONOUS LATTICE}

A preliminary lattice of a quasi-isochronous ring for a B Factory in the PEP tunnel has been designed by W. Corbett, M. Donald and A. Garren [5] The 43-meter-long cell in this design does not contain negative bends. It has the betatron tunes $\nu_{x}=0.75, \nu_{y}=0.25$, and can be obtained by a modification of three cells of a regular FODO lattice; see Fig. 1. 


\section{MINIMAL VALUE OF THE MOMENTUM COMPACTION FACTOR}

The value of $\alpha_{2}$ defines the lower limit for $\alpha_{1}$. Indeed, the longitudinal motion with $\alpha$ given by Eq. (1) is characterized by two stationary points: $\delta=0$ and $\delta=-\alpha_{1} / \alpha_{2}$. Which one of them is a stable point depends on the relative sign of $\alpha_{1}$ and $\alpha_{2}$. but the distance between the stationary points should be larger than the energy spread in the beam. Hence, the size of the RF bucket for the quasi-isochronous ring is limited by the condition $\delta<\left|\alpha_{1} / \alpha_{2}\right|$. For the PEP isochronous lattice [6], $\alpha_{2}=0.027$. Taking $\delta=0.005$, which corresponds to the $0.5 \%$ energy spread at injection and a safety factor of 8 to 10 in the ratio of the bucket size to the rms energy spread in the collision mode, we obtain the low limit [6] of $\alpha_{1}=1.35 \times 10^{-4}$. This magnitude of $\alpha$ is smaller then its value in the present design only by a factor of 7 (which could result in the reduction of the beam size by a factor of 2 to 3). Such a value of $\alpha_{s}$ has been discussed by M. Zisman [ $\bar{\imath}]$ for a B Factory with a regular lattice. Hence, the synchrotron tune in the practically interesting case is still large compared to the damping time: the transition energy is sufficiently small; and the longitudinal dynamics still may be described using the standard formalism. As was noticed by M. Zisman [7], reduction of the transverse emittance with $\alpha_{1}$ can be controlled by wigglers-implying that we do not consider the effect of small $\alpha_{1}$ on the transverse dynamics.

Another concern is time-dependent errors of the magnets. The $\alpha_{1}$ can, in principle, fluctuate in time due to ripples in the power supply and, if the design value of $\alpha_{1}$ is very small, $\alpha_{1}$ may change sign. We studied variations of $\alpha_{1}$ with the code MAD giving random variation of the quadrupole strength or the field in the bend magnets. With rms quad strength variations $0.1 \%$, we found the variations $\delta \alpha_{1} /\left\langle\alpha_{1}\right\rangle=12 \%$. Simultaneous variation of the dipole fields with rms $0.01 \%$ gives $\delta \alpha_{1} /\left\langle\alpha_{1}\right\rangle=16 \%$. Such variations of $\alpha_{1}$ are small and would not cause any problems.

\section{STABILITY OF COHERENT MOTION}

A study of the stability of coherent motion for a quasi-isochronous storage ring by Peliegrini and Robin [2], emphasizes the importance of including radiatiun damping in the stability condition. This modifies the threshold of the microwave longitudinal instability

$$
\frac{I_{a v}}{I_{A}}<\sqrt{\frac{\pi}{2}} \frac{Z_{0}}{(Z / n)_{\text {eff }}} \frac{\alpha_{1} \gamma \delta^{2} \sigma_{z}}{s_{B}} F(b)
$$

by the additional factor $F(b)$. Here $I_{A}=e c / r_{0}=1.7 \times 10^{4} \mathrm{~A}, Z_{0}=4 \pi / c=$ $377 \Omega$. $I_{a v}$ is the average beam current, $\sigma_{z}$ is rms bunch length, $s_{b}$ is the bunch spacing, and $Z_{\text {eff }}$ is the effective impedance for a harmonic number $n=\omega / \omega_{\text {rev }}$. The factor $F(b)$ is determined by the dispersion equation

$$
1=-i \Lambda J(a, b, d) \text {, }
$$

where

$$
\Lambda=\frac{I_{a v}}{I_{A}} \frac{(Z / n)_{e f f}}{Z_{0}} \sqrt{\frac{1}{\pi}} \frac{s_{B}}{\alpha_{1} \gamma \delta^{2} \sigma_{z}},
$$

and the dispersion integral $J(a, b, d)=J_{1}+i J_{2}$ depends on the unperturbed longitudinal distribution function $f$. For the Gaussian distribution $f(x)=e^{-x^{2}} / \sqrt{\pi}$

$$
J(a, b, d)=J_{1}+i J_{2}=\int_{-\infty}^{\infty} \frac{d x d f / d x}{a+i b+x+d \cdot x^{2}}
$$

where

$$
a=\frac{\nu_{n, R}}{\sqrt{2} n \delta \alpha_{1}}
$$

is dimensionless coherent tune shift,

$$
d=\frac{\alpha_{2} \delta \sqrt{2}}{\alpha_{1}},
$$

proportional to the nonlinear part $\alpha_{2}$ of the momentum compaction factor, and

$$
b=\frac{\nu_{n, I}}{n \delta \omega_{0} \alpha_{1} \sqrt{2}}
$$

defines decrement of the coherent mode, or damping of the $n$th harmonic of the distribution function $f_{n} \propto \exp \left(-\nu_{n, I} t\right)$. If the imaginary part of the tune shift comparable with the damping time $\zeta$ is allowed, $\nu_{n, I}=c(\zeta / 2)$, then for maximum

$$
n \omega_{0}=\frac{c}{\sigma_{z} \sqrt{2 \pi}}
$$

$b$ takes the form

$$
b=\frac{\sqrt{\pi} \zeta \sigma_{z}}{2 \alpha_{1} \delta} .
$$

Using the standard relationship between $\mathrm{rms} \sigma_{z}$ and the energy fluctuations per turn due to synchrotron radiation $\left\langle\delta^{2}\right\rangle_{\mathrm{tu}}$

$$
\sigma_{z}^{2}=\left(\frac{R \alpha_{1}}{\nu_{s}}\right)^{2} \frac{\left\langle\delta^{2}\right\rangle_{\mathrm{turn}}}{\zeta}
$$

it is easy to find that

$$
\sigma_{z} \propto \sqrt{\frac{\alpha_{1}}{e V_{0} \cos \phi_{s}}}
$$

and $b$ scales as $b \propto 1 / \sqrt{\alpha_{1} e V_{0} \cos \phi_{s}}$, where $e V_{0}$ is RF voltage per turn and $\phi_{s}$ is equilibrium RF phase ( $\phi_{s}=0$ without radiation losses). 
The factor $F(b)$ in Eq. (2) is given as $F(b)=1 / J_{2}$, with $a$ defined by the equation $J_{1}=0$

For the B Factory, we take the ratio of the rms $\delta$ to a bucket size $\alpha_{1} / \alpha_{2}$ equal to 10: then $d=\sqrt{2}=0.1414$. The function $F(b)$ is depicted in Fig. 2. For the LER parameters $\zeta=37 \mathrm{msec}, \delta=10^{-3}, \quad \sigma_{z}=1 \mathrm{~cm}$, and for $\alpha_{1}=1.5 \times 10^{-3}$, parameter $b=0.53$. This corresponds to the factor $F \simeq 2.0$ instead of $F=1 / \sqrt{2}$, if the damping is not taken into account $(b=0)$. Hence, the damping substantially increases the threshold current. Calculations given are for large $b$ scaling $F(b) \simeq$ $b^{\kappa}$, where $\kappa \simeq 2.5$ for large $b$; see Fig. 3 . The impedance for bunches that are short compared to the beam pipe radius, scales as $(Z / n)_{e f f} \propto \sigma_{z}^{3 / 2}$. In this case,

$$
I_{a v} \propto \frac{1}{\sigma_{z}\left(e V_{0} \cos \phi_{s}\right)^{\kappa-1}} .
$$

In the "broad band impedance" model, $(Z / n)_{\text {eff }} \propto \sigma_{z}^{2}$, giving

$$
I_{a v} \propto \frac{1}{\left(\sigma_{z} e V_{0}^{r} \cos \phi_{s}\right)^{\kappa-1}} .
$$

Thus, scaling down $\sigma_{z}$ with $\mathrm{RF}$ voltage fixed increases the threshold current for the same bunch length, provided $\kappa>1$. This is the case for sufficiently large $b$ (see Fig. 3). The threshold current increases with $b$, meaning that the same damping is more efficient for small $\alpha_{1}$. Another effect, dependence of the synchrotron tune on $\delta$ at a given $\alpha_{1}$, depends on $\alpha_{2}$. Comparing curves with two different values of the parameters $b, d$, it is easy to see that the energy spread of the synchrotron tune tends to increase the threshold current, but the effect is small. The deviation from a linear dependence $F \propto b$ is already noticable for $b \simeq 1$. The lower limit of the RF voltage is set by the radiation losses.

The coherent tune shift scales for maximum harmonic $n$ [Eq. (8)] as $a(b) / b$. The dependence $a(b)$ is practically linear; see Fig. 4 . Hence, $\nu_{n, R}$ is almost independent of $b$-changing from the value $\nu_{n, R}=-2.7 \times 10^{-2}$ at $\mathrm{b}=0.53$ (which corresponds to $\delta=10^{-3}, a_{1}=10^{-3}, R=35 \mathrm{Cm}, \sigma_{z}=1 \mathrm{~cm}$ ) to $\nu_{n, R}=$ $-1.1 \times 10^{-2}$ at very large $b \gg 1$.

The coherent tune shift for longitudinal coupled bunch instability scales in the Wang formalism as $\alpha_{1} / \nu_{s} \propto \sqrt{\alpha_{1}}$. Again, no additional problems should be expected with small $\alpha_{1}$.

It is worth noticing that the standard formalism describing instabilities might need some revision. Particularly for small $\nu_{s}$, the increment of the instability may be smaller then the synchrotron tune. In this case, instability should be considered as a fast, single-turn instability which may have unusual (not exponential) growth in time [8].

\section{CONCLUSION}

The threshold average current of a beam of short bunches moving in a quasiisochronous lattice may be substantially higher than that of a regular ring. It is worthwhile to design the lattice for the $\mathrm{B}$ factory to allow for change to the quasiisochronous lattice, as has been done by W. Corbett, M. Donald and A. Garren. Except perhaps ior the effect of synchro-betatron resonances, which will require some additional study and maybe tighter constraints on the energy spread at injection, the quasi-isochronous ring looks very promising. The field errors and fluctuation of the power supply do not cause major problems. However, short bunches may require a completely new design of the interaction region.

\section{ACKNOWLEDGMENTS}

I am grateful to $M$. Donald for information on the quasi-isochronous lattice for the PEP tunnel and to A. Hutton, S. Kheifets, R. Miller, R. Ruth and $D$. Pestrikov for useful discussions.

\section{REFERENCES}

1. S. Chattopadhyay et al., Proc. ICFA Workshop on Low Emittance $e^{-} e^{+}$ Beams (Brookhaven National Lab., 1987); also BNL 52090 (1987).

2. C. Pellegrini and D. Robin, Nucl. Instrum. Methods, A301, 27-36 (1991).

3. Perry B. Wilson, SLAC-PUB-2884 (1982).

4. "An Asymmetric B Factory Based on PEP," LBL-PUB-5303; SLAC-372 CALT-68-1715; UCRL-ID-106426; UC-IRPA-91, 01 (1991)

5. W. J. Corbett, M.H.R. Donald, and A. A. Garren, SLAC-PUB-5538, LBL-30669 (1991).

6. C. Pellegrini and D. Robin, unpublished.

7. M. S. Zisman, ABC Note-12, ESG Tech. Note-148 (1990).

8. A. V. Burov, N. S. Dikansky, and D. V. Pestrikov, in Proc. 13th Int. Conf. on High-Energy Accelerators, Novosibirsk, 1986, ed., A. N. Skrinsky (Nanka, 1987).

\section{DISCLAIMER}

This report was prepared as an account of work sponsored by an agency of the United States Government. Neither the United States Government nor any agency thereof, nor any of their employees, makes any warranty, express or implied, or assumes any legal liability or responsibility for the accuracy, completeness, or usefulness of any information, apparatus, product, or process disclosed, or represents that its use would not infringe privately owned rights. Reference herein to any specific commercial product, process, or service by trade name, trademark, manufacturer, or otherwise does not necessarily constitute or imply its endorsement, recommendation, or favoring by the United States Government or any agency thereof. The views and opinions of authors expressed herein do not necessarily state or reflect those of the United States Government or any agency thereof. 


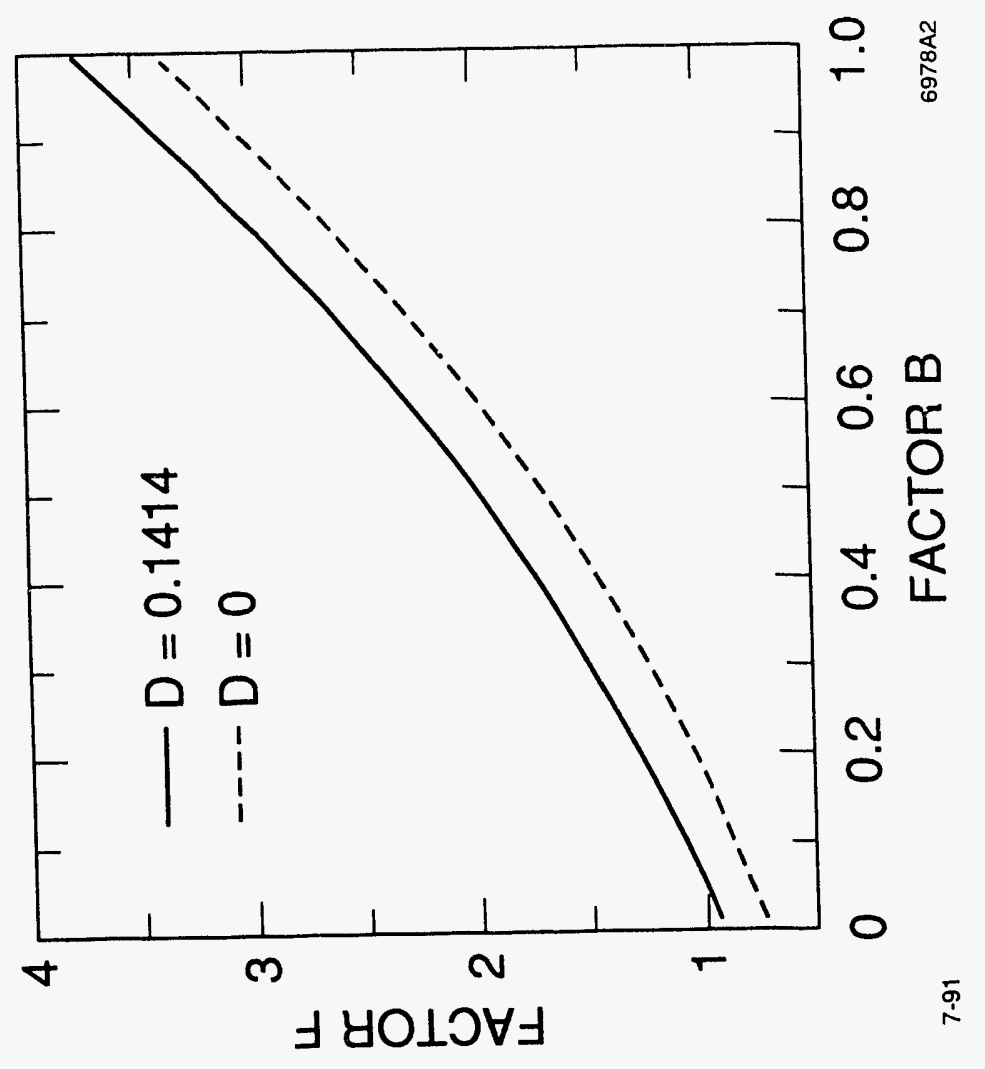

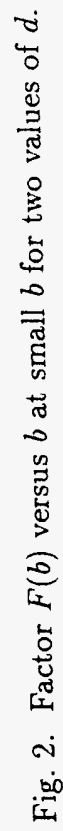

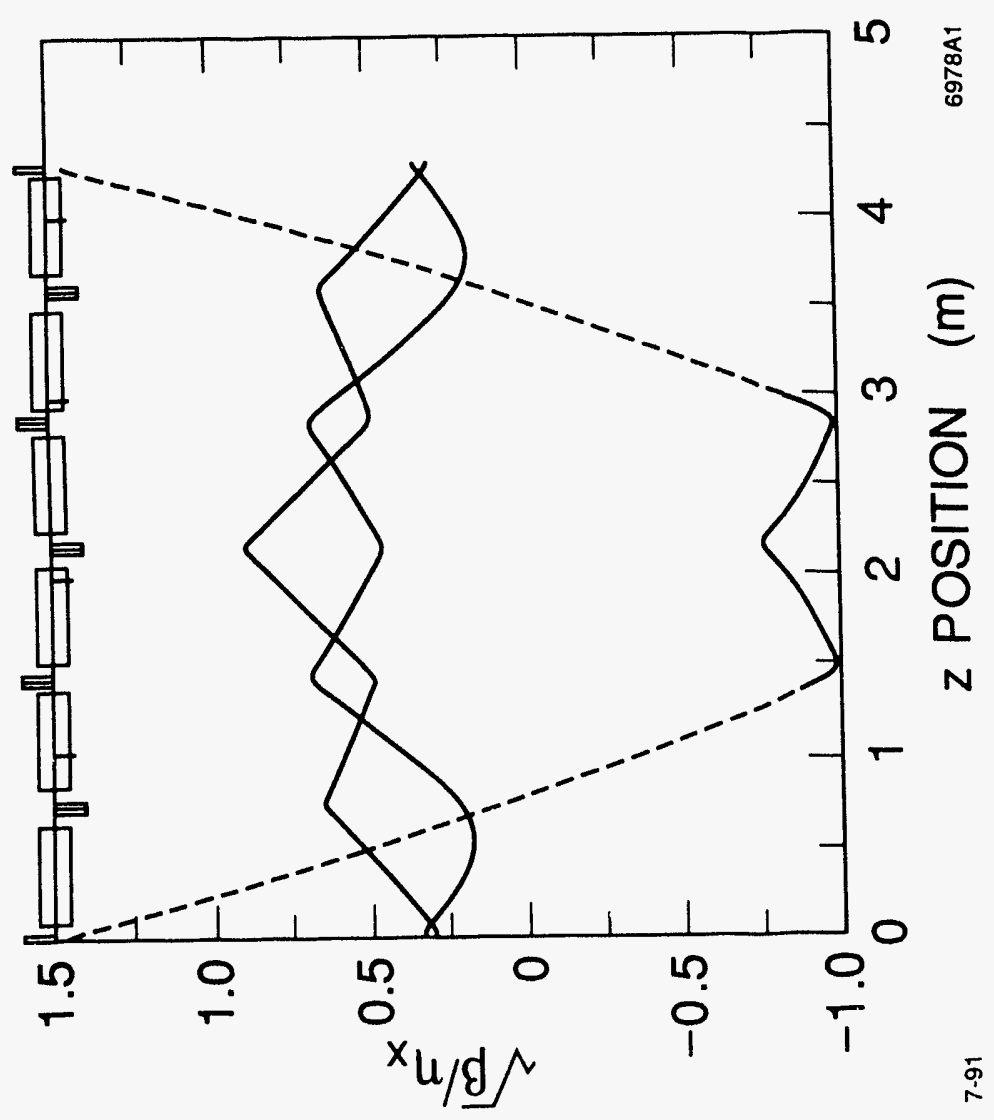

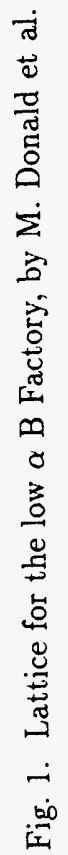




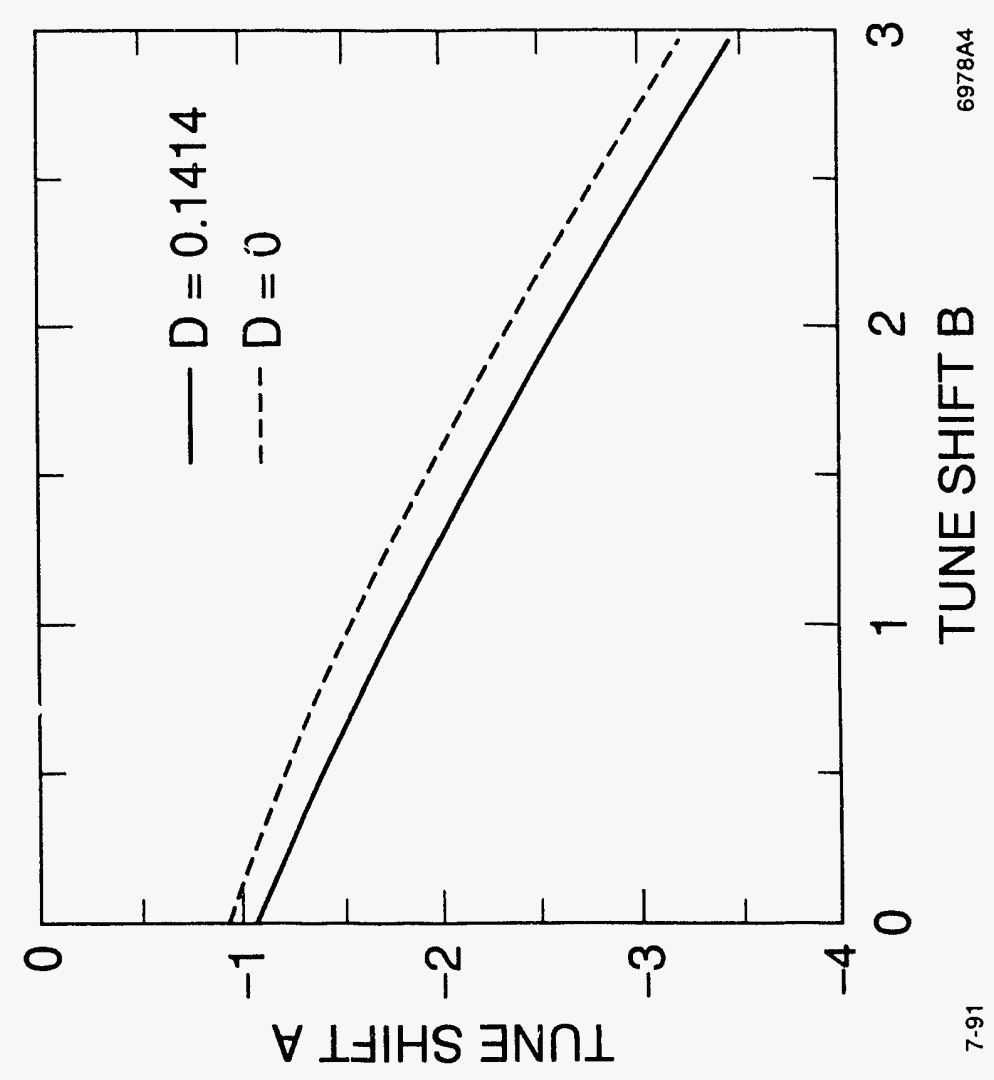

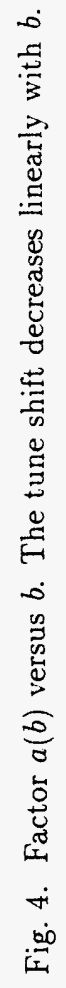

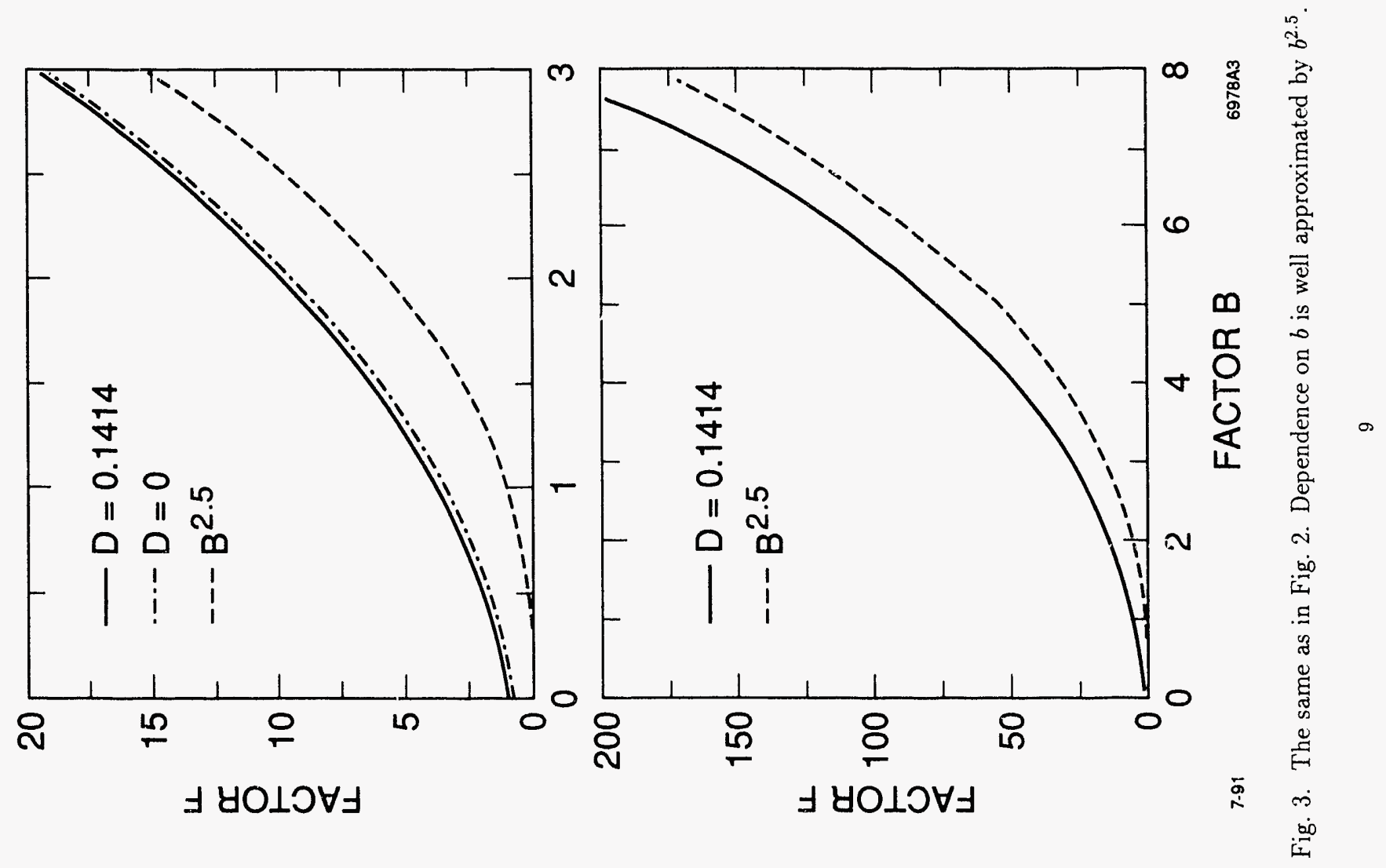



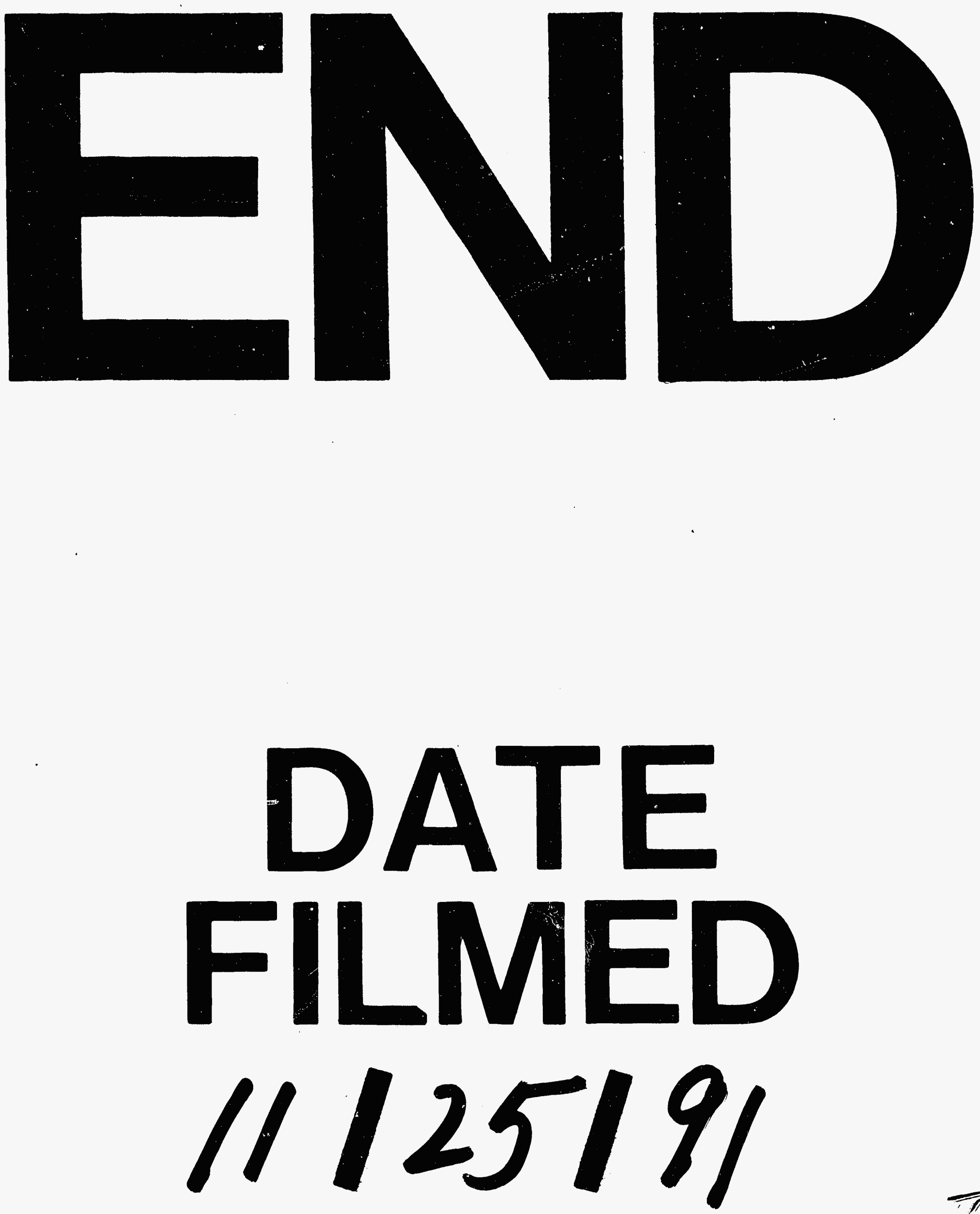

$\overline{1}$ 
$\|$ 